\title{
Correspondence:
}

\section{Clinical characteristics and plasma antibody titer of patients with COVID-19 in Zhejiang, China ${ }^{* \#}$}

\author{
Wei-ling XIANG ${ }^{1,3}$, Jing-jing CHENG ${ }^{1,3}$, \\ Lian-peng $\mathrm{WU}^{2}$, Bing-yu CHEN ${ }^{1,3}$, Wen-xin $\mathrm{LI}^{4}$, \\ Dan-ying QIU $^{3}$, Wei ZHANG ${ }^{3}$, Fei-hang GE$^{3}$, \\ Dong CHEN $^{\dagger 2,3}$, Zhen WANG Whi, $^{\dagger 1,3}$ \\ ${ }^{1}$ Research Center of Blood Transfusion Medicine, Ministry of \\ Education Key Laboratory of Laboratory Medicine, Department of \\ Blood Transfusion, Zhejiang Provincial People's Hospital, People's \\ Hospital of Hangzhou Medical College, Hangzhou 310014, China \\ ${ }^{2}$ Department of Laboratory Medicine, Wenzhou Central Hospital and \\ Sixth People's Hospital of Wenzhou, Wenzhou 325000, China \\ ${ }^{3}$ Rehabilitation \& Sports Medicine Research Institute of Zhejiang \\ Province, Zhejiang Provincial People's Hospital, People's Hospital of \\ Hangzhou Medical College, Hangzhou 310014, China \\ ${ }^{4}$ Research on the Application of Big Data-driven Integrated Blood \\ Collection and Supply Auxiliary Diagnosis and Treatment System, \\ Dongyang People's Hospital, Dongyang 322100, China \\ †E-mail: chendong_wz@126.com; wangzhen@hmc.edu.cn
}

https://doi.org/10.1631/jzus.B2000593

Coronavirus disease 2019 (COVID-19) is an infectious disease caused by severe acute respiratory syndrome coronavirus 2 (SARS-CoV-2), which first affected humans in China on December 31, 2019 (Shi et al., 2020). Coronaviruses generally cause mild, selflimiting upper respiratory tract infections in humans,

\footnotetext{
Corresponding authors

* Project supported by the Zhejiang Medical and Health Science and Technology Project (Nos. 2017KY005, 2019KY017, and 2018KY868), the Key Projects Jointly Constructed by the Ministry and the Province of Zhejiang Medical and Health Science and Technology Project (No. WKJ-ZJ-2019), and the Key and Major Projects of Traditional Chinese Medicine Scientific Research Foundation of Zhejiang Province (Nos. 2019ZZ001 and 2018ZY001), China

${ }^{\#}$ Electronic supplementary materials: The online version of this article (https://doi.org/10.1631/jzus.B2000593) contains supplementary materials, which are available to authorized users

(ib) ORCID: Wei-ling XIANG, https://orcid.org/0000-0001-5365-3951 (C) Zhejiang University and Springer-Verlag GmbH Germany, part of Springer Nature 2020
}

such as the common cold, pneumonia, and gastroenteritis (To et al., 2013; Berry et al., 2015; Chan et al., 2015). According to the Report of the World Health Organization (WHO)-China Joint Mission on COVID19 (WHO, 2020), the case fatality rate of COVID-19 increases with age, while the rate among males is higher than that among females $(4.7 \%$ and $2.8 \%$, respectively). Since an effective vaccine and specific anti-viral drugs are still under development, passive immunization using the convalescent plasma (CP) of recovered COVID-19 donors may offer a suitable therapeutic strategy for severely ill patients in the meantime. So far, several studies have shown therapeutic efficacy of CP transfusion in treating COVID19 cases. A pilot study first reported that transfusion of $\mathrm{CP}$ with neutralizing antibody titers above 1:640 was well tolerated and could potentially improve clinical outcomes through neutralizing viremia in severe COVID-19 cases (Chen et al., 2020). Immunoglobulin $\mathrm{G}(\mathrm{IgG})$ and $\mathrm{IgM}$ are the most abundant and important antibodies in protecting the human body from viral attack (Arabi et al., 2015; Marano et al., 2016). Our study aimed to understand the aspects of plasma antibody titer levels in convalescent patients, as well as assessing the clinical characteristics of normal, severely ill, and critically ill patients, and thus provide a basis for guiding CP therapy. We also hoped to find indicators which could serve as a reference in predicting the progression of the disease.

We used a colloidal gold test strip assay to determine the IgG and IgM antibody titers of CP samples collected from three hospitals (Huang et al., 2019), including Wenzhou Central Hospital (Sixth People's Hospital of Wenzhou) (139 cases), the First People's Hospital of Jiaxing City (20 cases), and Ningbo Huamei Hospital of the Chinese Academy of Sciences (134 cases). These patients were initially diagnosed by 
reverse transcription-polymerase chain reaction (RTPCR) via throat swab or sputum. The time of CP collection was approximately $7,14,21,28,35$, 42 , and $49 \mathrm{~d}$ after symptom onset. A commercial COVID-19 IgG/IgM Rapid Test Cassette produced by Zhejiang Orient Gene Biotech Co., Ltd. (Huzhou, China) was applied to detect the samples' stock solution and their dilutions. Each $\operatorname{IgG} / \mathrm{IgM}$ positive CP sample was diluted in saline at volume ratios of $1: 80$, $1: 160,1: 320,1: 640$, and 1:1280. We also retrospectively retrieved data on the epidemiological characteristics and clinical information of patients diagnosed with COVID-19 in Wenzhou Central Hospital as of February 24, 2020 from the electronic medical record system.

Of the $139 \mathrm{CP}$ samples from Wenzhou Central Hospital, 55 were collected within 4 weeks after symptom onset, 50 were collected within 4-6 weeks after symptom onset, and 34 were collected more than 6 weeks after symptom onset. These were mostly discontinuous samples, and we were fortunate to find four patients with continuous samples. We found that in the first month, the titer level of IgG was mainly between 80 and 160, rising to $160-320$ in the next 2 weeks and then to 320 or higher after 6 weeks (Table 1). There were four patients for whom we had enough consecutive blood samples from the first week (symptom onset) to the seventh week. We learned that IgG antibody titer levels of 320-640 or higher always appeared within 4-6 weeks after symptom onset (Fig. 1). This result was consistent with other studies showing that IgG reached higher levels after four weeks from the onset of COVID-19 symptoms (Li et al., 2020; Long et al., 2020), which can thus be considered the best time to donate plasma. However, many samples still also showed positive IgM titer results in this period, which should be taken into consideration in screening plasma donor. IgM is a serologic marker which usually represents a recent or current infection, and low IgM levels may not be required for $\mathrm{CP}$ donor selection if available donors are limited (Singh et al., 2015; Samanta and Willis, 2016; Zhang et al., 2020). Positive IgM samples were obtained from a total of 260 COVID-19 convalescent patients from three hospitals, including Wenzhou Central Hospital (139 total cases, 86 men and 53 women), the First People's Hospital of Jiaxing City (13 total cases, 10 men and 3 women), and Ningbo Huamei Hospital (108 total cases, 55 men and 53 women). The IgM positivity rate was higher among men and the difference by sex was statistically significant $(P<0.05)$. This result may be due to pre-existing fitness and health levels or other factors like differences in immunoregulation between male and female, which needs further research. We discovered correlations between IgG/IgM and different laboratory indices such as C-reactive protein (CRP), prothrombin time (PT), absolute value of lymphocyte count (LYM), albumin (ALB), lactic dehydrogenase (LDH), and hemoglobin (HGB), which are shown in Fig. S1. The IgM titer in CP had a positive correlation with $\mathrm{CRP}$ and $\mathrm{PT}$, but a negative correlation with LYM and ALB. Meanwhile, the IgG titer in CP had a positive correlation with CRP and $\mathrm{LDH}$, but a negative correlation with LYM and HGB $(P<0.05)$. These indices are related to the degree of inflammation, immunologic or coagulation function. In addition, the IgM titer in $\mathrm{CP}$ shared the same growth trend with $\mathrm{CRP}$ and $\mathrm{PT}$, which supports the idea that patients with persistent $\operatorname{IgM}$ may have a shorter duration of positive RT-PCR, resulting in clinical conditions that worsened less compared to patients without the presence of anti-SARS-CoV-2 IgM antibodies (Lee et al., 2020).

In our study, 13 patients were severely or critically ill patients reported in Wenzhou Central Hospital, aged 37-77 years, with a mean age of 56.4 years. Eleven of these 13 patients were male $(84.6 \%)$ and two were female $(15.4 \%)$, which may indicate that

Table 1 Antibody titer levels from COVID-19 patients' plasma (discontinuous) in different stages of disease progress in Wenzhou Central Hospital of Zhejiang Province $(n=139)$

\begin{tabular}{cccc}
\hline \multirow{2}{*}{ Titer level } & $\begin{array}{c}\text { IgG-positive case (within } \\
\text { 4 weeks after symptom onset) }\end{array}$ & $\begin{array}{c}\text { IgG-positive case (within } \\
\text { 4-6 weeks after symptom onset) }\end{array}$ & $\begin{array}{c}\text { IgG-positive case (more than } \\
\text { 6 weeks after symptom onset) }\end{array}$ \\
\hline 80 & 20 & 8 & 3 \\
160 & 21 & 22 & 10 \\
320 & 13 & 16 & 17 \\
640 & 1 & 4 & 4 \\
\hline
\end{tabular}




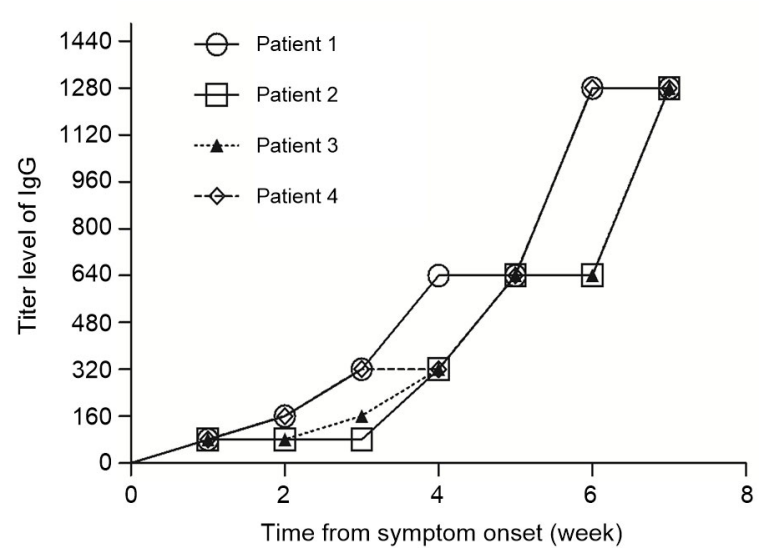

Fig. 1 Plasma antibody titer levels of consecutive convalescent plasma from convalescent patients at Wenzhou Central Hospital of Zhejiang Province $(n=4)$

middle-aged men tend to experience worse symptoms from the virus. The reported routes of transmission for these severely and critically ill patients included living in the epidemic area (69.2\%), a history of contact with a COVID-19 patient in public (15.4\%), or close contact with a COVID-19-positive family member (15.4\%), supporting the importance of quarantine measures such as wearing masks, washing hands, and disinfecting frequently to reduce the risk of transmission. Seven of the 13 patients $(53.8 \%)$, including two $(50.0 \%)$ of the critically ill and five $(55.6 \%)$ of the severely ill patients, presented with gastrointestinal symptoms such as diarrhea, nausea, and vomiting. On admission, other frequent symptoms included fever in $12(92.3 \%)$, cough in 11 $(84.6 \%)$, dyspnea in eight $(61.5 \%)$, and fatigue in eight $(61.5 \%)$ patients. What calls for special attention is that presented symptoms represent multiple systems such as the digestive tract and the respiratory tract, which makes it difficult to distinguish COVID19 from other diseases with overlapping symptoms.

As Table 2 shows, among the 13 severe and critically ill patients, eight $(61.5 \%)$ patients were found to have increased serum creatine kinase levels, including six severely ill and two critically ill patients. Eleven patients $(84.6 \%)$, including four of the critically ill patients $(100.0 \%)$ and seven of the severely ill patients $(77.8 \%)$, showed elevated serum lactate dehydrogenase levels. Of the 13 patients, eight patients (61.5\%) showed decreased serum potassium levels, including three of the critically ill patients $(75.0 \%)$, and five of the severely ill patients $(55.6 \%)$. Also, eight $(61.5 \%)$ patients presented reduced blood calcium concentrations, including two of the critically ill patients $(50.0 \%)$ and six of the severely ill patients (66.7\%), suggesting that electrolyte disturbances occur during the process of the disease. It is necessary to address any water and electrolyte metabolism disorders in time to avoid heart failure and prevent or mitigate liver and kidney dysfunctions.

Meanwhile, we must also pay attention to patients with complicated courses of the diseases. The absolute value of lymphocytes was decreased in 12 (92.3\%) patients, including four $(100.0 \%)$ of the critically ill and eight $(88.9 \%)$ of the severely ill patients, although the degree of decline was higher among the former. CRP levels were increased for all patients. CRP is a very sensitive indicator of an acute-phase reaction and is commonly used in clinic to identify the existence of co-infection. When the body is resistant to infection, or following infection, the function of the systemic immune system is weak and shows a poor ability to resist bacteria. This highlights the fact that it is important to actively work to prevent hospital-related infections, such as the occurrence of ventilator-associated pneumonia or tube infections. To supply oxygen to severely ill patients, nasal catheter oxygenation and mask oxygenation were used, while none were given noninvasive/ invasive ventilator oxygenation. Either mask oxygen or nasal catheter oxygen can meet the body's oxygen demands in severely ill patients. All four critically ill patients required noninvasive ventilator oxygenation $(100.0 \%)$. The latest research has found that the mortality rate of COVID-19 patients with acute respiratory distress syndrome (ARDS) is $50 \%-70 \%$ (Liu et al., 2020). Early noninvasive positive-pressure ventilation should be deployed as conditions allow; this can increase the body's oxygen supply and improve prognosis by facilitating the recovery of lung function during rehabilitation.

There are several limitations in this study. The sample size was small, limited to Zhejiang Province, and lacked a sufficient quantity of consecutive samples. Because of this, we were not able to carry out a large-scale, well-designed clinical trial and the result may not be representative of the large target populations. More samples and subjects should be included in similar studies in the future to explore the correlation of antibody levels with disease severity. 
Table 2 Epidemiology and clinical characteristics of severely and critically ill COVID-19 patients at Wenzhou Central Hospital of Zhejiang Province

\begin{tabular}{|c|c|c|c|}
\hline Characteristics & Total $(n=13)$ & Severe disease $(n=9)$ & Critical illness $(n=4)$ \\
\hline \multicolumn{4}{|l|}{ Epidemiological data } \\
\hline Male patient & $11(84.6 \%)$ & $9(100.0 \%)$ & $2(50.0 \%)$ \\
\hline Female patient & $2(15.4 \%)$ & $0(0 \%)$ & $2(50.0 \%)$ \\
\hline Age (year) & $56.4(37.0-77.0)$ & $57.7(46.0-77.0)$ & $54.5(37.0-71.0)$ \\
\hline History of exposure to the epidemic area & $9(69.2 \%)$ & $6(66.7 \%)$ & $3(75.0 \%)$ \\
\hline History of contact with an infected patient & $2(15.4 \%)$ & $2(22.2 \%)$ & $0(0 \%)$ \\
\hline No obvious history of contact with an infected patient & $2(15.4 \%)$ & $1(11.1 \%)$ & $1(25.0 \%)$ \\
\hline Days to diagnosis (d) & $6.5(3.0-17.0)$ & $9.4(3.0-17.0)$ & $5.3(3.0-7.0)$ \\
\hline Fever & $12(92.3 \%)$ & $8(88.9 \%)$ & $4(100.0 \%)$ \\
\hline Fatigue & $8(61.5 \%)$ & $7(77.8 \%)$ & $1(25.0 \%)$ \\
\hline Cough & $11(84.6 \%)$ & $7(77.8 \%)$ & $4(100.0 \%)$ \\
\hline Chest distress & $1(7.6 \%)$ & $0(0 \%)$ & $1(25.0 \%)$ \\
\hline Dyspnea & $8(61.5 \%)$ & $4(44.4 \%)$ & $4(100.0 \%)$ \\
\hline Diarrhea & $7(53.8 \%)$ & $5(55.6 \%)$ & $2(50.0 \%)$ \\
\hline Nausea and vomiting & $7(53.8 \%)$ & $5(55.6 \%)$ & $2(50.0 \%)$ \\
\hline \multicolumn{4}{|l|}{ Laboratory test (reference value) } \\
\hline White blood cell $\left(\times 10^{9} \mathrm{~L}^{-1}\right)(4-10)$ & $4.5(2.4-8.8)$ & $4.3(2.4-8.8)$ & $5.0(3.8-7.6)$ \\
\hline Decreased white blood cell & $8(61.5 \%)$ & $6(66.7 \%)$ & $2(50.0 \%)$ \\
\hline Leukomonocyte $\left(\times 10^{9} \mathrm{~L}^{-1}\right)(1.1-3.2)$ & $0.7(0.1-1.0)$ & $0.6(0.3-1.0)$ & $0.5(0.1-0.8)$ \\
\hline Decreased leukomonocyte & $12(92.3 \%)$ & $8(88.9 \%)$ & $4(100.0 \%)$ \\
\hline Creatine kinase (U/L) (55-170) & $359.4(31.0-1933.0)$ & $229.4(31.0-273.7)$ & $603.3(80.0-1933.0)$ \\
\hline Increased creatine kinase & $8(61.5 \%)$ & $6(66.7 \%)$ & $2(50.0 \%)$ \\
\hline C-reactive protein $(\mathrm{mg} / \mathrm{L})(<8)$ & $47.2(22.8-101.9)$ & $43.4(22.8-96.2)$ & $55.87(36.6-101.9)$ \\
\hline Increased C-reactive protein & $13(100 \%)$ & $9(100.0 \%)$ & $4(100.0 \%)$ \\
\hline Lactic dehydrogenase (U/L) (114-240) & $298.1(196.0-457.0)$ & $265.0(196.0-384.0)$ & $372.8(295.0-457.0)$ \\
\hline Increased lactic dehydrogenase & $11(84.6 \%)$ & $7(77.8 \%)$ & $4(100.0 \%)$ \\
\hline Potassium (mol/L) (3.5-5.5) & $3.42(2.76-4.39)$ & $3.58(3.40-4.39)$ & $3.06(2.76-3.76)$ \\
\hline Decreased potassium & $8(61.5 \%)$ & $5(55.6 \%)$ & $3(75.0 \%)$ \\
\hline Serum calcium $(\mathrm{mol} / \mathrm{L})(2.05-2.60)$ & $1.80(0.93-2.29)$ & $1.70(0.93-2.29)$ & $2.03(1.98-2.08)$ \\
\hline Decreased serum calcium & $8(61.5 \%)$ & $6(66.7 \%)$ & $2(50.0 \%)$ \\
\hline \multicolumn{4}{|l|}{ Oxygen/respiratory support } \\
\hline Nasal catheter for oxygen & $11(84.6 \%)$ & $8(88.9 \%)$ & $3(75.0 \%)$ \\
\hline Mask oxygen & $4(30.7 \%)$ & $0(0 \%)$ & $4(100.0 \%)$ \\
\hline Noninvasive ventilator support & $4(30.7 \%)$ & $0(0 \%)$ & $4(100.0 \%)$ \\
\hline Invasive ventilator support & $0(0 \%)$ & $0(0 \%)$ & $0(0 \%)$ \\
\hline
\end{tabular}

Data are expressed as number (percentage) or average (range)

In conclusion, IgG antibody titer levels of 320 640 or higher always appeared within $4-6$ weeks after symptom onset, which indicates that this is the best time to collect $\mathrm{CP}$. At that point, some patients still showed positive IgM titer results in the plasma. The positive rate of IgM in CP tended to be higher among males than among females. Critically ill patients needed to rely on noninvasive ventilation for survival. Early noninvasive positive-pressure ventilation should be deployed as conditions allow in order to reduce the mortality rate. Almost all severe and critical patients had water and electrolyte metabolism disorders, which indicates that attending physicians need to pay more attention to this issue and take intervention measures as soon as possible. The incidence of coagulation function disorder and inflammation in the early stages of the disease can influence the antibody titer of $\operatorname{IgG}$ and $\mathrm{IgM}$ in $\mathrm{CP}$, which may help to screen appropriate 
CP donors in advance. COVID-19 patients were at risk of concurrent infections due to their compromised immune systems. The antibody profile has clear links to some clinical lab indexes (lymphopenia, CRP, and creatine), which may lay a foundation for future therapy.

\section{Contributors}

Wei-ling XIANG and Jing-jing CHENG conducted the testing. Lian-peng WU, Fei-hang GE, Dong CHEN, and Wei ZHANG collected the clinical data. Bing-yu CHEN, Wen-xin LI, and Dan-ying QIU performed the statistics and analyses of the data. Wei-ling XIANG wrote the first draft of the manuscript. Dong CHEN and Zhen WANG designed the study. All authors have read and approved the final manuscript and, therefore, have full access to all of the data in the study and take responsibility for the integrity and accuracy of the data.

\section{Acknowledgments}

We thank Dr. Ke HAO (Research Center of Blood Transfusion Medicine, Ministry of Education Key Laboratory of Laboratory Medicine, Department of Blood Transfusion, Zhejiang Provincial People's Hospital, People's Hospital of Hangzhou Medical College, Hangzhou, China) for revising manuscript and giving good suggestions.

\section{Compliance with ethics guidelines}

Wei-ling XIANG, Jing-jing CHENG, Lian-peng WU, Bing-yu CHEN, Wen-xin LI, Dan-ying QIU, Wei ZHANG, Fei-hang GE, Dong CHEN, and Zhen WANG declare that they have no conflict of interest.

All procedures followed were in accordance with the ethical standards of the responsible committee on human experimentation (institutional and national) and with the Helsinki Declaration of 1975, as revised in 2008 (5). Informed consent was obtained from all patients for being included in the study.

\section{References}

Arabi Y, Balkhy H, Hajeer AH, et al., 2015. Feasibility, safety, clinical, and laboratory effects of convalescent plasma therapy for patients with Middle East respiratory syndrome coronavirus infection: a study protocol. SpringerPlus, 4:709. https://doi.org/10.1186/s40064-015-1490-9

Berry M, Gamieldien J, Fielding BC, 2015. Identification of new respiratory viruses in the new millennium. Viruses, 7(3):996-1019. https://doi.org/10.3390/v7030996

Chan JFW, Yao YF, Yeung ML, et al., 2015. Treatment with lopinavir/ritonavir or interferon- $\beta 1 \mathrm{~b}$ improves outcome of MERS-CoV infection in a nonhuman primate model of common marmoset. J Infect Dis, 212(12):1904-1913. https://doi.org/10.1093/infdis/jiv392
Chen L, Xiong J, Bao L, et al., 2020. Convalescent plasma as a potential therapy for COVID-19. Lancet Infect Dis, 20(4): 398-400. https://doi.org/10.1016/S1473-3099(20)30141-9

Huang DQ, Chen R, Wang YQ, et al., 2019. Development of a colloidal gold-based immunochromatographic strip for rapid detection of Rice stripe virus. J Zhejiang Univ-Sci B (Biomed \& Biotechnol), 20(4):343-354. https://doi.org/10.1631/jzus.B1800563

Lee YL, Liao CH, Liu PY, et al., 2020. Dynamics of antiSARS-CoV-2 IgM and IgG antibodies among COVID-19 patients. $J$ Infect, 81(2):E55-E58. https://doi.org/10.1016/j.jinf.2020.04.019

Li L, Tong XL, Chen HW, et al., 2020. Characteristics and serological patterns of COVID-19 convalescent plasma donors: optimal donors and timing of donation. Transfusion, 60(8):1765-1772. https://doi.org/10.1111/trf.15918

Liu YL, Sun WW, Li J, et al., 2020. Clinical features and progression of acute respiratory distress syndrome in coronavirus disease 2019. medRxiv, preprint. https://doi.org/10.1101/2020.02.17.20024166

Long QX, Liu BZ, Deng HJ, et al., 2020. Antibody responses to SARS-CoV-2 in patients with COVID-19. Nat Med, 26(6):845-848. https://doi.org/10.1038/s41591-020-0897-1

Marano G, Vaglio S, Pupella S, et al., 2016. Convalescent plasma: new evidence for an old therapeutic tool? Blood Transfus, 14(2):152-157. https://doi.org/10.2450/2015.0131-15

Samanta D, Willis E, 2016. Focal seizure associated with human parvovirus B19 infection in a non-encephalopathic child. World J Pediatr, 12(1):118-120. https://doi.org/10.1007/s12519-015-0060-0

Shi Y, Wang G, Cai XP, et al., 2020. An overview of COVID-19. J Zhejiang Univ Sci B (Biomed \& Biotechnol), 21(5):343360. https://doi.org/10.1631/jzus.B2000083

Singh L, Mishra S, Prasanna S, et al., 2015. Seroprevalence of TORCH infections in antenatal and HIV positive patient populations. Med J Armed Forces India, 71(2):135-138. https://doi.org/10.1016/j.mjafi.2014.12.009

To KKW, Hung IFN, Chan JFW, et al., 2013. From SARS coronavirus to novel animal and human coronaviruses. $J$ Thorac Dis, 5(S2):S103-S108. https://doi.org/10.3978/j.issn.2072-1439.2013.06.02

WHO, 2020. Report of the WHO-China Joint Mission on coronavirus disease 2019 (COVID-19). https://www.who. int/publications/i/item/report-of-the-who-china-joint-miss ion-on-coronavirus-disease-2019-(covid-19)

Zhang LB, Pang RR, Xue X, et al., 2020. Anti-SARS-CoV-2 virus antibody levels in convalescent plasma of six donors who have recovered from COVID-19. Aging, 12(8):65366542 . https://doi.org/10.18632/aging.103102 


\section{List of electronic supplementary materials}

Fig. S1 Correlation between $\operatorname{IgG} / \operatorname{IgM}$ titer and different laboratory indexes

\section{中文概要}

题 目: 浙江省新冠病毒肺炎患者的临床特征和血浆抗体 滴度的调查与分析

目 的: 利用胶体金快速检测试剂盒测定恢复期患者血浆 中 $\mathrm{IgG} / \mathrm{IgM}$ 的抗体滴度, 回顾性分析新型冠状病 毒肺炎（COVID-19）患者临床特征、抗体效价 变化特点, 以及两者间的相关性。

创新点: 利用胶体金检测试剂盒检测恢复期血浆抗体滴 度，结合重症及危重症患者病例特点及实验室检 测指标分析血浆抗体效价变化规律，及其临床特 征的关系, 为临床重症及危重症患者诊治以及篮 选恢复期血浆供者提供参考依据。
方 法: 收集 293 份来自 COVID-19 康复者随访过程中的 血液标本, 取血浆原液, 用生理盐水按不同比例 (1:80、1:160、1:320 和 $1: 640)$ 稀释后进行胶体 金试纸法 $\operatorname{IgG} / \mathrm{IgM}$ 抗体检测, 并记录相应患者检 验指标水平。通过电子病历系统检索回顾性分析 温州市第六人民医院截至 2020 年 2 月 24 日确诊 的 COVID-19 患者的流行病学特征和临床信息。 通过线性回归分析两变量之间的相关性, $P<0.05$ 时具有统计学意义。

结 论: 症状出现 4 6 周时, COVID-19 患者恢复期血浆 $\mathrm{IgG}$ 滴度水平可达到 1:320 1:640 或者更高水平。 此时部分患者的血浆 IgM 仍为阳性。COVID-19 患者恢复期血浆 IgM 抗体滴度检测结果显示男 性较女性高。本研究中危重症患者均需要无创通 气来维持生命, 且重症和极危重症患者均易发生 水、电解质紊乱。COVID-19 发病早期发生凝血 功能障碍及炎症, 与恢复期血浆中 $\operatorname{IgG}$ 和 $\operatorname{IgM}$ 的 抗体滴度水平存在一定相关性。

关键词：新型冠状病毒肺炎（COVID-19）；恢复期血浆； 临床特征; 抗体滴度 